\title{
IRREDUCIBLE DIAGRAMS IN LANDAU-GINZBURG FIELD THEORY
}

\author{
T.A. WITTEN, Jr. ${ }^{1}$ \\ Physics Department, University of Michigan, Ann Arbor, MI 48109, USA
}

Received 16 March 1981

\begin{abstract}
It is shown that the free energy $W$ of a Landau-Ginzburg-Wilson field theory with $\mathrm{O}(n)$ symmetry may be written in terms of the generating function $V$ of diagrams irreducible in both propagator and interaction lines. This generalizes and simplifies a recent result of Des Cloizeaux. The functions $W$ and $V$ are related by a type of Legendre transformation on the bare mass variable.
\end{abstract}

The thermodynamics of a system near a second-order phase transition can often be expressed in terms of a field theory with an $n$-vector field $\phi$ representing the order parameter, and a lagrangian (Landau-Ginzburg-Wilson energy [1])

$$
\begin{aligned}
L([\boldsymbol{\phi}], u, \boldsymbol{H})=\int \mathrm{d}^{d} r & {\left[\frac{1}{2}(\underline{\nabla} \boldsymbol{\phi}(r))^{2}+\frac{1}{2} g u|\boldsymbol{\phi}(r)|^{2}\right.} \\
& \left.+\frac{1}{8} g \int \mathrm{d}^{d} r^{\prime}|\boldsymbol{\phi}(r)|^{2} v\left(r-r^{\prime}\right)\left|\boldsymbol{\phi}\left(r^{\prime}\right)\right|^{2}-\boldsymbol{\phi}(r) \cdot \boldsymbol{H}\right],
\end{aligned}
$$

where $v\left(r^{\prime}-r\right)$ is a short-ranged interaction normalized to unity, $\boldsymbol{H}$ is an external source, and the bare mass squared is represented as $g u$. This note shows how the free energy for this system may be written in terms of especially simple perturbation diagrams, namely those irreducible in both propagator and interaction lines.

The partition function for such a theory, $Z(u, H)$, is defined as

$$
Z(u, H) \equiv \int^{\prime} \mathrm{d}[\phi] \mathrm{e}^{-L},
$$

where the functional integration $\int^{\prime}$ contains an ultraviolet cutoff mechanism corresponding to the fluctuations of smallest spatial extent in the system-typically those of atomic scale. Full thermodynamic information is then contained in the free energy [2] $W(u, H)$ defined as $\ln Z(u, H)$.

\footnotetext{
' Supported by US National Science Foundation under DMR801-2867, Polymer Program.
} 
The diagrammatic expansion of $W(H)$ contains all connected diagrams. The same physical information may be expressed in terms of a simpler subset of diagrams, namely those "one propagator irreducible" (1PI) graphs which cannot be separated into two pieces by cutting a single propagator line. The sum $\Gamma(u, M)$ of these IPI graphs turns out to be simply the Legendre transform [3] of $W$ :

$$
\Gamma(u, M)=-W(u, H)+H M, \quad \text { where } M \equiv \partial W / \partial H .
$$

(In zero-loop order $\left.\Gamma(u, M)=\left.L([\phi], u, H)\right|_{\phi=M ; H=0}.\right)$

It is suggestive to look for an analogous relation for the diagrams which are irreducible in interaction lines as well as in propagators. Such a relation is derived below. The sum $V$ of these diagrams is essentially a Legendre transform of $\Gamma(u, M)$, but new features appear which are not present in the $W \rightarrow \Gamma$ transformation.

It is useful to express the free energy in terms of this function $V$, since one deals with a smaller number of diagrams than otherwise. More importantly, these diagrams are less singular than the $\Gamma$ diagrams. In the regime of spontaneous symmetry breaking, where $M$ remains non-zero as $H \rightarrow 0$, the loop expansion for $\Gamma$ fails because of the long-range transverse field correlations. But the loop expansion of $V$ is well behaved in this regime, as Schäfer and Horner have recently shown [4]. (Indeed many features of the present derivation are implicit in this work and a subsequent paper [5].) Des Cloizeaux [6] has shown the connection between $\Gamma$ and $V$ for the limit of a zero-component field, by another method.

The connection between $\Gamma$ and $V$ could be derived by taking a limit of a more general problem, with a second field $\psi$ in addition to the field $\phi$. In this formulation the interaction term $\phi^{2} v \phi^{2}$ would be simulated by a local interaction of the form $\phi^{2} \psi$. The original $v\left(r^{\prime}-r\right)$ emerges as a Yukawa interaction due to exchange of the $\psi$ field. The present derivation avoids this approach and requires no auxiliary field $\psi$. The aim is to make the derivation as perspicuous and economical as possible from the point of view of a condensed matter theorist.

The proof relies on the topological properties of the $\Gamma$ diagrams. A diagram for $-\Gamma$ may be represented as a skeleton expansion in the form of tree diagrams consisting of nodes connected by wavy interaction lines representing $-g$ factors (fig. 1a). Each node (shown as a box) with, say, $k$ interaction lines emerging is the sum of all diagrams which are one-interaction and one propagator irreducible (1IPI) and which have $k$ mass insertions. I denote the sum of such diagrams as the "node function" $V^{(k)}(M)$. This means that $V^{(1)}$ contains a zero-loop contribution $\frac{1}{2} M^{2}$. In these diagrams I have expanded out the mass term $g u$ explicitly; thus the propagators are massless. This also means that in the skeleton expansion some interaction lines simply terminate in $u$ factors.

The goal here is to find an algebraic expression relating $-\Gamma(u, M)$ to the node functions $V^{(k)}(M)$. This is made simpler by taking a derivative. The full $\partial(-\Gamma) / \partial n$ has itself a simple skeleton expansion. This is true because the $n$-dependence of a 


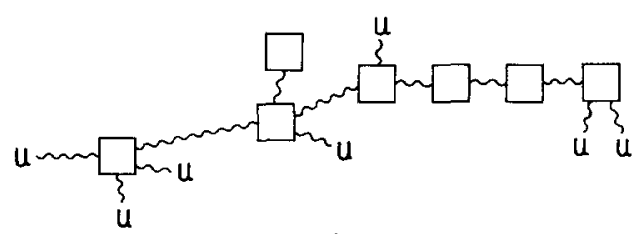

a.

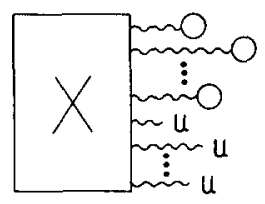

b.

Fig. 1. Skeleton diagrams for the function $\Gamma$. Wavy lines represent interaction factors $-g$; circles represent the sum of diagrams irreducible in propagator lines (1PI); boxes represent the sum of diagrams irreducible in propagator and interaction lines (1IPI). (a) shows a generic diagram for $\Gamma$; (b) shows one for $\partial \Gamma / \partial n$.

$-\Gamma$ diagram arises solely from the nodes $V^{(k)}(M)$ within it. (There is a factor $n$ for every loop of $\phi$ propagators.) The skeleton diagrams for $\partial(-\Gamma) / \partial n$ are formed from those of $-\Gamma$ itself by differentiating each $V^{(k)}(M)$ in turn. Denoting the differentiated $V^{(k)}(M)$ by a box with an $\times$, I draw the general skeleton diagram for $\partial(-\Gamma) / \partial n$ as in fig. $1 b$. Here the circles contain all $-\Gamma$ diagrams with a mass insertion: hence, each circle with its wavy line has the value $\partial(-\Gamma) / \partial u$. The skeleton diagrams for $\partial(-\Gamma) / \partial n$ may thus be summarized in an algebraic form:

$$
(\partial(-\Gamma) / \partial n)_{u, M}=\sum_{k=0}^{\infty} \frac{1}{k !} \frac{\partial V^{(k)}(M)}{\partial n}\left(-g u-\frac{\partial \Gamma}{\partial u}\right)^{k} .
$$

This may be simplified further by defining the generating function $V(w, M)$ :

$$
V(w, M) \equiv \sum_{k=0}^{\infty}(1 / k !) V^{(k)}(M)(-w)^{k} .
$$

Now the $-\Gamma$ derivative may be expressed

$$
(\partial(-\Gamma) / \partial n)_{u, M}=(\partial V / \partial n)_{w, M}, \quad w=g u+\partial \Gamma / \partial u ;
$$

i.e. the transformation $-\Gamma(u) \rightarrow V(w)$ preserves derivatives in the auxiliary variable $n$. This is the fundamental property of the Legendre transform. It is shown below that with minor modifications $\Gamma$ and $V$ are Legendre transforms. 
First I modify $\Gamma$ so that $w$ and $u$ are true conjugate variables. I define

$$
\Delta(u, M) \equiv \Gamma(u, M)+\frac{1}{2} g u^{2} .
$$

Then from eq. (6) $w=\partial \Delta / \partial u$, and $(\partial(-\Delta) / \partial n)_{u, M}=(\partial V / \partial n)_{w, M}$, as before. This means that $\Delta$ and $V$ are Legendre transforms as far as their $n$ dependence is concerned. By a parallel argument one may show that $\Delta$ and $V$ are Legendre transforms as far as their $M$ dependence is concerned:

$$
(\partial(-\Delta) / \partial M)_{u}=(\partial V / \partial M)_{w} .
$$

The result follows because all the $M$ dependence of a $-\Gamma$ diagram arises through its nodes $V^{(k)}(M)$, as with the $n$ dependence.

Since $V$ has the same $n$ and $M$ derivatives as the Legendre transform of $\Delta$, the two must be equal up to a function independent of $M$ and $n$ :

$$
V(w, M)=[-\Delta(u, M)+u w]+f(w) .
$$

To determine the function $f$, it suffices to choose a particular $M$ and $n$. One may take $M=n=0$, so that there are no diagrams with $\phi$ loops [7] and none with $M$ factors: both $\Gamma(u)$ and $V(w)$ then vanish. Comparing with eq. (7) one readily finds that $f=-w^{2} /(2 g)$. Thus $\left[V+w^{2} /(2 g)\right]$ and $\left[\Gamma+\frac{1}{2} g u^{2}\right]$ are Legendre transforms. This gives the desired connection between IIPI diagrams and the free energy $W$ of the system.

The diagrams for $V(w)$ may be simplified further. The quantity $V(w)$ depends on $w$ only through mass insertions in the $V^{(k)}$. Thus all the $w$ dependence may be included by shifting from massless propagators to propagators $G(p, w)$ with mass $w^{1 / 2}: G(p, w)=1 /\left(p^{2}+w\right)$. The $V(w)$ diagrams are then identically that subset of $-\Gamma$ diagrams which are one-interaction irreducible, where propagator lines denote factors of $G(p, w)$.

This connection between IIPI diagrams and the free energy may likely be extended to include position dependent source fields [1] $H, u, M$, and $w$.

I am grateful to L. Schäfer, R. Akhouri and R. Savit for helpful suggestions. This problem was suggested by M. Mirkovitch.

\section{References}

[1] E. Brézin, J.C. Le Guillou and J. Zinn-Justin, in Phase transitions and critical phenomena, vol. 6, eds.

C. Domb and M.S. Green (Academic, 1976), p. 202, eq. (9.1)

[2] Ibid. eq. (2.13)

[3] Ibid. eqs. (2.15), (2.18)

[4] L. Schäfer and H. Horner, Z. Phys. B29 (1978) 251

[5] A. Knoll, L. Schäfer and T.A. Witten, J. de Phys. 42 (1981) 767

[6] J. Des Cloizeaux, J. de Phys. 41 (1980) 749

[7] P.G. De Gennes, Phys. Lett. 38A (1972) 339 